\title{
Success rate of resuscitation after out-of-hospital cardiac arrest
}

Anthony MH Ho ${ }^{1}$, FRCPC, FCCP, Glenio B Mizubuti ${ }^{~ *}$, MSc, MD, Adrienne $\mathrm{K} \mathrm{Ho}^{2}, \mathrm{MB}, \mathrm{BS}$, Song Wan ${ }^{3}$, MD, FRCS, Devin Sydor ${ }^{1}$, MD, FRCPC, David C Chung ${ }^{4}$, MD, FRCPC

\author{
${ }^{1}$ Department of Anesthesiology and Perioperative Medicine, Queen's University, Canada \\ ${ }^{2}$ Department of Oncology, The Christie NHS Foundation Trust, Manchester, United Kingdom \\ ${ }^{3}$ Division of Cardiac Surgery, Department of Surgery, The Chinese University of Hong Kong, Shatin, Hong Kong \\ ${ }^{4}$ Department of Anaesthesia and Intensive Care, The Chinese University of Hong Kong, Shatin, Hong Kong
}

*Corresponding author: gleniomizubuti@hotmail.com

Hong Kong Med J 2019;25:254-6

https://doi.org/10.12809/hkmj187596

A recent study in Hong Kong documented the low success rate of resuscitation after adult out-of-hospital cardiac arrest (OHCA). Survival to hospital discharge with good neurological outcome was $1.5 \% .{ }^{1} \mathrm{~A}$ median delay of 12 minutes for defibrillation was one factor that contributed to poor outcomes. ${ }^{1}$ In ventricular fibrillation or pulseless ventricular tachycardia, every minute without defibrillation drastically reduces the chance of successful resuscitation. ${ }^{2}$ To partially mitigate the delayed arrival of trained personnel to administer defibrillation, ${ }^{1}$ widespread deployment of automated external defibrillators (AEDs) in public places in conjunction with public education is a worthwhile strategy. ${ }^{3}$

A 10-year review reported the incidence of $\mathrm{OHCA}$ in Hong Kong children to be 5.37/100000 person-years. ${ }^{4}$ Most causes were respiratory (for which immediate action should be assessment and management of Airway, Breathing, and Circulation), and only $9.4 \%$ of individuals had a shockable rhythm. Survival with good neurological outcome was $13.2 \%{ }^{4}$

Cardiopulmonary resuscitation (CPR) is immediately required while awaiting defibrillation, or when defibrillation is unsuccessful, when the arrest is of respiratory origin (some 6\% of adult $\mathrm{OHCAs}$ and the majority of paediatric arrests ${ }^{4}$ ) or if rhythm is not shockable. The goal of CPR is to oxygenate the heart and brain until a life-sustaining rhythm and spontaneous circulation can be restored. Traditionally, CPR started with rescue breathing followed by breathing and chest compression cycles. However, the current untrained bystander CPR protocol includes compression-only CPR (CO-CPR) with no rescue breathing. The reduced emphasis on traditional rescue breathing aims to increase bystander participation and avoid hyperventilation, and is based in part on recognition that the blood oxygen level is sufficient during the first few minutes of abrupt ventricular fibrillation or pulseless ventricular tachycardia (although this may not apply when the cardiac arrest is secondary to respiratory causes). Importantly, it enables chest compression to start immediately and without interruption, a lack of which is linked to poor outcomes. ${ }^{5}$ However, because $60 \%$ of adult and $38 \%$ of paediatric OHCAs in Hong Kong are unwitnessed, ${ }^{1,4}$ these first few minutes of sufficient oxygen in the blood (for sudden arrest of cardiac origin) may have already elapsed. This raises the question of whether a one-size-fitsall bystander CPR with no breathing component is advisable. It is not surprising that the rates of survival with good neurological outcomes are low. Until recently, the Hong Kong emergency medical services did not provide over-the-phone instructions on bystander CPR, thus precluding the incorporation of a breathing component (even when ventilation is clearly necessary) prior to the arrival of trained personnel. Since last year, however, Hong Kong has established a dispatcher instruction hotline which should hopefully allow improved guidance for untrained bystanders to provide better quality CPR.

There thus exists a conflict: the need to promptly initiate chest compression that should be uninterrupted, and the need to oxygenate that itself may delay and interrupt chest compression. By promoting CO-CPR for untrained bystanders in OHCA, the International Liaison Committee on Resuscitation has apparently chosen to assign a lower priority to arrests that are unwitnessed and those of respiratory origin without acknowledging that there are two components of rescue breathing: airway patency and mouth-to-mouth breathing. It is only the mouth-to-mouth breathing component that deters public participation, causes hyperventilation, and delays and interrupts chest compression. We have argued that the need for respiration and for prompt uninterrupted chest compression can both be accommodated if the mouth-to-mouth breathing only is removed, but not the airway patency component. $^{6}$

The simple manoeuvre that healthcare personnel routinely use to open the airway of a comatose patient is the chin lift, with or without insertion of an oropharyngeal airway. The chin lift elevates the hyoid bone and in turn the epiglottis by means of the hyoid-epiglottic ligament. It also 


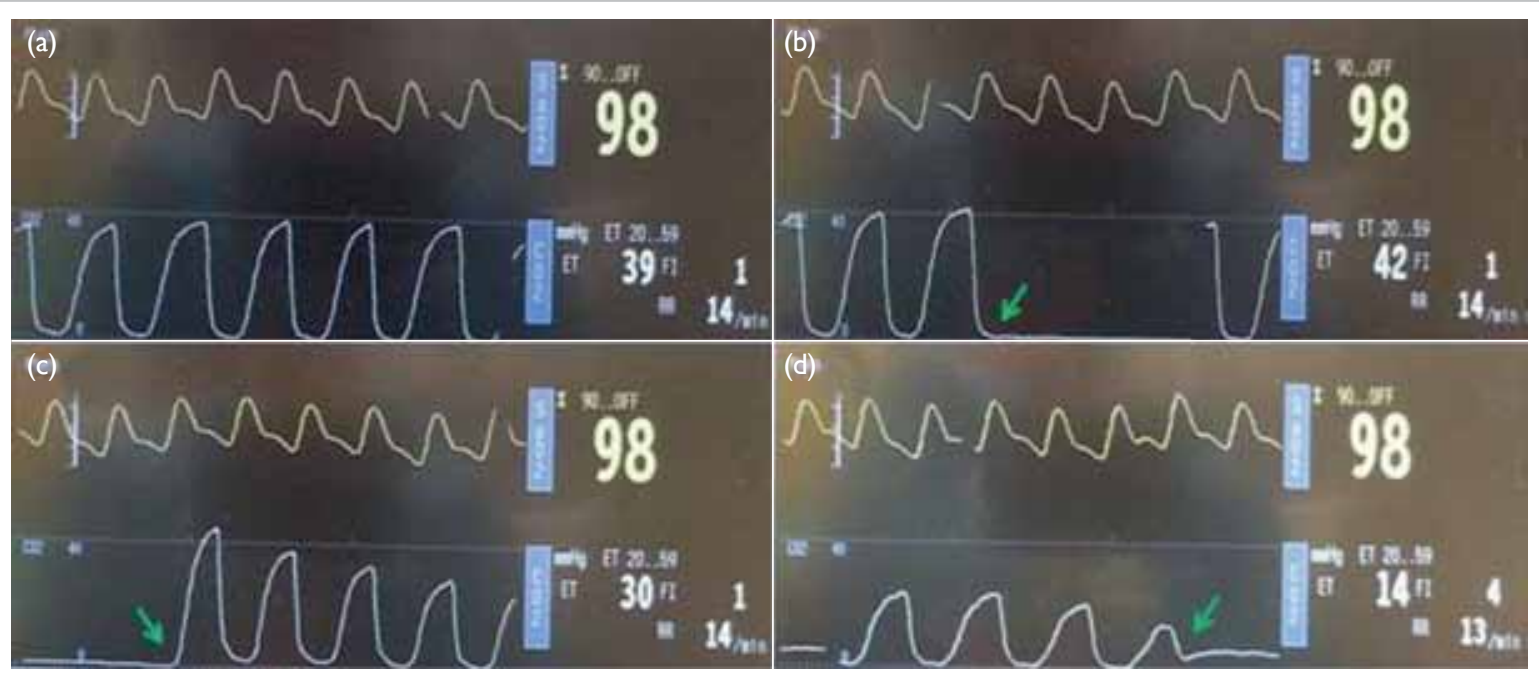

FIG. Exhaled carbon dioxide (bottom tracing) sampled at the nostrils of a deeply sedated patient with no obstructive sleep apnoea with (a) chin lift alone, (b) head sequentially transitioned to the neutral position, (c) head turned $45^{\circ}$ to the side, and (d) head returned to the neutral position. The green arrow represents the transition points in head position. Although the chin lift consistently and greatly improves airway patency, the improvement with the head turn is usually less consistent and less dramatic

prevents the tongue from being sucked into the path of airflow during inhalation. Both chin lift and the oropharyngeal airway are extremely effective. With a patent airway, gasping, which is associated with improved survival, ${ }^{7}$ and passive ventilation from chest compression ${ }^{8,9}$ are facilitated. Passive ventilation via an orotracheal tube during CPR has been shown to be superior to CPR with bag-mask ventilation. ${ }^{10}$ When airway patency is maintained using chin lift or an oropharyngeal airway, passive ventilation should theoretically have a similar beneficial effect. Chin lift is feasible when there is a second bystander. For single-bystander CPR, a less reliable technique is to turn the patient's head as far as possible to one side before promptly commencing uninterrupted CPR. In anaesthetised or deeply sedated patients with obstructive sleep apnoea, the head turn may reduce airway obstruction. ${ }^{11,12}$ The Figure illustrates the effectiveness of chin lift and head turn in maintaining airway patency in a healthy deeply sedated patient. From experience, a head turn is less consistent or efficient in opening the airway but given its simplicity, should be performed immediately prior to the commencement of uninterrupted CO-CPR by a single bystander.

Strategies for increasing OHCA survival include widespread deployment of AEDs in public places and education campaigns. It has been recently proposed that oropharyngeal airways across the age spectrum be included in public AED boxes. ${ }^{13}$ An instruction sheet with illustrations on how to size an oropharyngeal airway should be included for quick reference. The airway can be inserted by a second bystander while the primary rescuer is applying the defibrillator pads or performing CO-CPR. If only one bystander is present, the oropharyngeal airway can be inserted immediately prior to commencing uninterrupted CO-CPR. Because more than half of adult OHCAs in Hong Kong occur in the home, ${ }^{1}$ the government should consider providing one size $9(9 \mathrm{~cm}$, yellow $)$ and one size $8(8 \mathrm{~cm}$, green $)$ oropharyngeal airways for all households and dwellings with a resident who is an elderly person or has cardiac problems; these two airway sizes are suitable for almost all men and women, respectively. ${ }^{14}$ Consideration should also be given to providing paediatric oropharyngeal airways for households with a seriously ill child, who comprise a significant proportion of paediatric OHCAs in Hong Kong. ${ }^{4}$

Out-of-hospital cardiac arrest is a serious public health problem worldwide. Despite great effort and expense (eg, therapeutic hypothermia ${ }^{15}$ ), improvements in meaningful survival after cardiac arrest have been only marginal. The steps and strategies we have suggested may have a large impact at extremely low cost. Hong Kong should take a lead in exploring ways to improve OHCA outcomes by incorporating airway patency and passive ventilation in the untrained bystander CPR protocol, running public education and training campaigns, and conducting observational (eg, before-after) and randomised controlled studies to validate the concept.

\section{Author contributions}

All authors had full access to the data, contributed to the study, approved the final version for publication, and take responsibility for its accuracy and integrity.

Concept and design of study: AMH Ho, GB Mizubuti. Acquisition of data: AMH Ho, GB Mizubuti, D Sydor. Analysis or interpretation of data: AMH Ho, GB Mizubuti. Drafting of the manuscript: AMH Ho, GB Mizubuti, AK Ho. 
Critical revision for important intellectual content: D Sydor, S Wan, DC Chung.

\section{Conflicts of interest}

All authors have disclosed no conflicts of interest.

\section{Funding/support}

This research received no specific grant from any funding agency in the public, commercial, or not-for-profit sectors.

\section{References}

1. Fan KL, Leung LP, Siu YC. Out-of-hospital cardiac arrest in Hong Kong: a territory-wide study. Hong Kong Med J 2017;23:48-53.

2. Chan PS, Krumholz HM, Nichol G, Nallamothu BK; American Heart Assocation National Registry of Cardiopulmonary Resuscitation Investigators. Delayed time to defibrillation after in-hospital cardiac arrest. N Engl J Med 2008;358:9-17.

3. Weisfeldt ML, Sitlani CM, Ornato JP, et al. Survival after application of automatic external defibrillators before arrival of the emergency medical system: evaluation in the resuscitation outcomes consortium population of 21 million. J Am Coll Cardiol 2010;55:1713-20.

4. Law AK, Ng MH, Hon KL, Graham CA. Out-of-hospital cardiac arrest in the pediatric population in Hong Kong: a 10 -year review at a university hospital. Pediatr Emerg Care 2018;34:179-84.

5. Brouwer TF, Walker RG, Chapman FW, Koster RW. Association between chest compression interruptions and clinical outcomes of ventricular fibrillation out-of-hospital cardiac arrest. Circulation 2015;132:1030-7.

6. Ho AM, Mizubuti GB, Wan S. Improving the success rate of chest compression-only CPR by untrained bystanders in adult out-of-hospital cardiac arrest: maintaining airway patency may be the way forward. Anesth Analg 2018;126:351-3.

7. Debaty G, Labarere J, Frascone RJ, et al. Long-term prognostic value of gasping during out-of-hospital cardiac arrest. J Am Coll Cardiol 2017;70:1467-76.

8. Safar P, Brown TC, Holtey WJ. Failure of closed chest cardiac massage to produce pulmonary ventilation. Dis Chest 1962;41:1-8.

9. Deakin CD, O'Neill JF, Tabor T. Does compressiononly cardiopulmonary resuscitation generate adequate passive ventilation during cardiac arrest? Resuscitation 2007;75:53-9.

10. Bobrow BJ, Ewy GA, Clark L, et al. Passive oxygen insufflation is superior to bag-valve-mask ventilation for witnessed ventricular fibrillation out-of-hospital cardiac arrest. Ann Emerg Med 2009;54:656-62.

11. Safiruddin F, Koutsourelakis I, de Vries N. Upper airway collapse during drug induced sleep endoscopy: head rotation in supine position compared with lateral head and trunk position. Eur Arch Otorhinolaryngol 2015;272:485-8.

12. Walsh JH, Maddison KJ, Platt PR, Hillman DR, Eastwood PR. Influence of head extension, flexion, and rotation on collapsibility of the passive upper airway. Sleep 2008;31:1440-7.

13. Ho AK. Should oropharyngeal airways be included with public automatic external defibrillators? Circulation 2018;138:1620-2.

14. Kim HJ, Kim SH, Min JY, Park WK. Determination of the appropriate oropharyngeal airway size in adults: Assessment using ventilation and an endoscopic view. Am J Emerg Med 2017;35:1430-4.

15. Moler FW, Silverstein FS, Holubkov R, et al. Therapeutic hypothermia after in-hospital cardiac arrest in children. $\mathrm{N}$ Engl J Med 2017;376:318-29. 